Praktikabilität von der richterlichen Kontrolle bereits im Gesetzgebungsverfahren

(vom späteren Vollzug ganz zu schweigen) übrig bleiben wird, kann sich jeder, der den parlamentarischen Betrieb kennt, leicht ausmalen.

Unerwähnt bleibt im AE die politische Kontrolle der Polizei. Gerade weil im Polizeibereich aufgrund der sofortigen Durchführung polizeilicher Maßnahmen Rechtsschutz häufig nicht mehr effektiv zu erlangen ist, gewinnt diese Problematik eine große Bedeutung. Zu denken wäre dabei vor allem an einen unabhängigen "Polizeibeauftragten « ${ }^{16}$. Diskussionswürdig - wenn auch schwer normierbar - erscheint eine Verpflichtung, unter bestimmten Voraussetzungen unabhängige Untersuchungsausschüsse einzusetzen ${ }^{17}$. Zu denken wäre auch an die Umgestaltung der polizeilichen Leitungsstruktur in Richtung auf den politisch verantwortlichen und dementsprechend jederzeit ablösbaren Leiter jeder einzelnen Abteilung.

Insgesamt gesehen ist der mit dem AE unternommene Schritt zur Initiierung einer neuen öffentlichen Diskussion der weiteren Entwicklungsrichtung unseres Polizeirechts notwendig gewesen und zu begrüßen. Unfreiwillig hat das Professorenteam aber auch vielen bisher gesetzlich nicht geregelten polizeilichen Tätigkeiten ein erstes legitimatorisches Stützkorsett verpaßt. Da hätte man sich gewünscht, daß dieses Mieder so geschneidert worden wäre, daß wirklich weder oben noch unten noch zwischen den Schnüren das viele überflüssige Fett der gegenwärtigen Praxis herausquillt und dann die Ministerialbürokratie im Gesetzgebungsverfahren die ganze Sache mit einem Griff wieder aufnesteln kann.

Klaus Groth

\title{
Republikanischer Anwaltsverein gegründet
}

Wie in der Kritischen Justiz 1978, S. I 82 berichtet, hatte der 2. Deutsche Strafverteidigertag im April 1978 den Rechtsanwalt Dr. Werner Holtfort beauftragt, die Gründung eines neuen Anwaltsvereins vorzubereiten. Der »Aufruf zur Gründung einer bundesweiten Anwaltsvereinigung zur Verteidigung der freien Advokatur« (abgedruckt Kritische Justiz 1979, S. I1 1/2) war von 33 Juristen unterzeichnet, von Sozialdemokraten, einigen Freidemokraten, Parteilosen und zwei CDU-Mitgliedern. Es fanden sich darunter die Unterschriften der Rechtsanwälte Peter Becker (Marburg), Manfred Coppik, Werner Holtfort, Waldemar Klischies, Rudolf Monnerjahn, Wolfgang Pennigsdorf, Rudolf Schöfberger, Gerhard Schröder, Michael Sexauer und der Hochschullehrer Wolfgang Däubler, Erhard Denninger, Gerald Grünwald, Senatsdirektor Reinhard Hoffmann, Ulrich Klug, Ulrich Preuß, Fritz Sack und Hans-Peter Schneider. Dem Aufruf folgten I 8 Rechtsanwälte, die am Io.

I6 Ein solcher könnte jedenfalls mit Sicherheit sachgerechter Uberwachungsfunktionen wahrnehmen, als der vom AE PolG in $\$ 46$ Abs. 3 (in sehr eingeschränktem Umfang) mit solchen Funktionen betraute Datenschutzbeauftragte.

17 Solche Untersuchungsausschüsse haben sich in der Berliner Polizeigeschichte - obwohl nicht gesetzlich vorgesehen und jedesmal gegen den Willen staatlicher Stellen von privater Seire eingesetzt - häufig sehr positiv ausgewirkt. So konnte der sogenannte "Ermittlungsausschuß $\beta_{1967}$ einiges zur Aufhellung der Yorgänge um den Tod des Studenten Benno Ohnesorg beitragen; weitere Ausschüsse halfen bei der Aufklärung von Vorgängen anläßlich des Todes von Georg v. Rauch, der "Akstion Wasserschlag* (vgl. dazu Jacobs, KJ 2/1977, S. i7s ff.) sowie neuestens bei der Untersuchung des polizeilichen Vorgehens gegen eine »antifaschistische Demonstration in Berlin-Tempelhof (vgl. Berliner Tagesspiegel, is. März 1979, S. 14: "Hübner zum Knüppeleinsatz«). 
und I I. Februar 1979 in Hannover den "Republikanischen Anwaltsverein e. V.» gründeten.

Bereits der Gründungsaufruf spiegelte die Motivation der in Hannover versammelten Rechtsanwälte wider. In ihm wurde hingewiesen auf den fortschreitenden Abbau von Freiheitsrechten der Bürger, auf die sich häufenden gesetzlichen Einschränkungen von Schutzrechten angeklagter Bürger, auf die Tendenz, die Anwaltschaft beamtenähnlich an den Staat zu binden. Es wurde zugleich festgestellt, daß es bisher keine bundesweite Anwaltsvereinigung gäbe, die sich diesen gefährlichen Entwicklungen entschieden genug entgegengestellt habe. Infolgedessen müsse eine Organisation von Rechtsanwälten geschaffen werden, die nicht - wie die bestehenden Standesvertretungen - Standesinteressen vertrete, sondern die die Beistandsfunktion des Rechtsanwalts für den Bürger gegen staatlichen und wirtschaftlichen Machtanspruch fördere.

Entsprechend dem ursprünglich erteilten Auftrag war zur Gründung einer allgemeinen Anwaltsvereinigung aufgerufen worden. Demgegenüber strebte ein Teil der Kollegen lediglich die Gründung einer bundesweiten Strafverteidiger-Vereinigung an. Deshalb wandte sich Rechtsanwalt Zimmermann für weitere 40 Kolleginnen und Kollegen aus Nordrhein-Westfalen am 3. 2. 1979 mit einem Rundbrief an die interessierten Kollegen, in dem er ausführte:

"Bei den vorbereitenden Diskussionen hat es unterschiedliche Auffassungen darüber gegeben, ob die Vereinigung als Strafverteidiger-Vereinigung oder als allgemeine Anwaltsvereinigung konzipiert werden sollte. Wir wissen aus einer Vielzahl von regionalen Treffen und aus den Diskussionen der Kollegen, die sich bundesweit regelmäßig in Frankfurt getroffen haben, daß die überwiegende Meinung dahin geht, daß nur eine Strafverteidiger-Vereinigung zur Zeit sinnvoll ist. Das Bedürfnis der meisten Kollegen geht dahin, auf dem Gebiet des Straf- und Strafprozeßrechts die eigene Meinung organisiert zur Geltung bringen zu können und für diesen Zweck eine feste Organisationsstruktur zu schaffen. Das Gewicht einer Strafverteidiger-Vereinigung würde auch darauf beruhen, daß wir einen wesentlichen Teil der auf diesem Gebiet engagiert arbeitenden Kollegen zusammenfassen könnten und damit einen relevanten Teil der Strafverteidiger repräsentieren würden. Gegem die Gründung einer allgemeinen Anwaltsvereinigung spricht, daß auf den übrigen Rechtsgebieten außerhalb der Strafverteidigung noch kein solcher inhaltlicher Konsens besteht. Weder ist die Rechtsentwicklung auf diesen Gebieten mit der im Bereich des Strafprozeßrechts zu vergleichen, noch haben wir bisher auf diesen Gebieten in entsprechender Weise zusammengearbeitet und entsprechende Positionen erarbeitet. Wir würden deshalb auf diesen Rechtsgebieten keine ausgewiesenen Positionen vertreten können und auch nicht einen relevanten Teil von Kollegen, die auf diesen Gebieten arbeiten, repräsentieren.«

Mit den in dem Rundbrief vorgetragenen Argumenten setzten sich die in Hannover versammelten Anwaltskollegen am Vormittag des 10. 2. I 979 auseinander, nachdem Rechtsanwalt Dr. Werner Holtfort in seinem Eröffnungsreferat darauf u. a. entgegnet hatte:

"Schwerer fiel die Entscheidung über Anwalts- oder bloße Strafverteidiger-Vereinigung, die an 3 Tagungen sehr ernsthaft erwogen wurde. Aber auch hierüber konnten wir uns verständigen, und zwar im Sinne einer Vereinigung, die alle Rechtsanwälte umfassen könne. Gewiß sollen die bestehenden örtlichen Strafverteidiger-Vereinigungen ihre erfolgreiche Arbeit fortsetzen und die Gründung weiterer Strafverteidiger-Vereinigungen fördern. Die Gründung aber lediglich einer bundesweiten Strafverteidiger-Vereinigung hätte zur Folge, daß ein erhebliches Potential wie die Vereinigungen "Freie Advokatur " und "Bund freier Rechtsanwälte « sowie alle Kollegen, die sich nicht vorwiegend als Strafverteidiger begreifen, von der Mitarbeit ausgeschlossen wären. Auch könnte die Arbeit einer bloßen Strafverteidiger-Vereinigung nicht als legitimiert angesehen werden, Gebiete wie das Ausländerrecht, das Arbeitsrecht, den Zugang zum öffentlichen Dienst, das Polizeirecht, Atomrecht, die Chancengleichheit im Recht auch für die soziale Unterschicht oder auch des Standesrechtes der Rechtsanwälte zu bearbeiten und auf diesen Gebieten mitzusprechen. Es kann nicht übersehen werden, daß auch die Betätigungsfreiheit derjenigen Kollegen, die vorwiegend nicht auf strafrechtlichem Gebiet tätig sind, einschneidenden Restriktionen unterliegen. Die neu zu gründende Vereinigung muß es 
sich deshalb zur Aufgabe machen, jegliche anwaltliche Tätigkeit von jedem staatlichen Eingriff und jeder staatlichen Definition freizuhalten. Sie muß daher vom Anspruch und von der Qualifikation der Mitglieder her befugt sein, sich auf all den genannten Rechtsgebieten kompetent zu äußern.«

Viele der angereisten Kollegen, die sich nicht ausschließlich mit Strafverteidigung befassen, brachten in der Diskussion zum Ausdruck, daß nur eine bundesweite allgemeine Anwaltsvereinigung im Sinne des Gründungsaufrufs wirksam tätig werden könne. Gerade die Anwaltskollegen aus der Provinz befürchteten, nur wegen des Umstands, keine reine Strafverteidigerpraxis zu betreiben, weiterhin von einer Beteiligung und Repräsentation ausgeschlossen zu sein. Tatsächlich betreffen ja die im Gründungsaufruf zum Ausdruck gekommenen Besorgnisse nicht allein das Feld der Strafverteidigung. Ein Abbau von Rechtsstaatlichkeit ist vielmehr handfest und aktuell spürbar im zivilen und öffentlichen Recht. Dies wurde von der Gruppe um Rechtsanwalt Zimmermann letztlich nicht bestritten. Schwerpunkt der Diskussion bildete deshalb die Frage, ob die bundesweite, auf Strafverteidiger beschränkte Organisation gegenüber der geplanten und tatsächlich gegründeten bundesweiten allgemeinen Anwaltsvereinigung wirksamer arbeiten könnte. Die Vertreter dieser These haben sich nicht durchgesetzt. Nachdem sie in einer Abstimmung unterlegen waren, reisten sie daher - etwa 40 Kollegen, insbesondere aus Nordrhein-Westfalen - schon am ersten Tag ab, ohne an den weiteren Diskussionen über die Gründung teilzunehmen.

Ein Problem war weiterhin das Verhältnis der neuen Vereinigung zum Deutschen Anwaltsverein. Dazu sagte Rechtsanwalt Holtfort in seinem Eingangsreferat:

$\leadsto$ Die Zugehörigkeit unserer Vereinigung wird die zum DAV von uns aus nicht ausschließen. Wir suchen keine Feindschaft zum DAV. Auf manchem Gebiete wird man gut zusammenarbeiten, auf anderen sich ergänzen können. Das dringliche Bedürfnis nach einer freiheitlich gesinnten neuen Anwaltsvereinigung hat sich durch die entweder fehlende oder halbherzige Politik des DAV in der Sicherung von Grund- und Freiheitsrechten der Staatsbürger ergeben. Zur Praxis der Berufsverbote z. B. hat der DAV ebenso geschwiegen wie zur Reform des $\$ 218$, des Scheidungs-, Versammlungs- oder des Kriegsdienstverweigerungsrechtes. Die Verteidigerausschließung durch staatliche Gerichte, das Verbot der Mehrfachverteidigung, die Trennscheibe, die Ưberwachung des Schriftverkehrs zwischen Untersuchungsgefangenem und Verteidiger, das Kontaktsperregesetz und ähnliches sind sämtlich mit Zustimmung des DAV ergangen in dessen Annahme, sie seien für einen freiheitlichen Rechtsstaat gerade eben noch hinnehmbar. Das charakterisiert den Unterschied: der DAV gibt sich mit einer Minimalisierung der Grundrechte, der Bürgerschutzrechte und der Freiheit der Advokatur zufrieden; wir sind auf eine Maximalisierung aus. Der DAV mag weiterhin Standesinteressen vertreten. Wir wollen die Beistandsfunktion des Rechtsanwalts für den Bürger verwirklichen, und zwar auch für den durch staatlichen und wirtschaftlichen Machtanspruch bedrohten. Nach meiner Úberzeugung setzen wir damit das Freiheits- und Grundrechteprogramm des Grundgesetzes gegenüber einer auf vordemokratischem Verständnis beruhenden Anwaltstradition durch. Zwei Kollegen haben mir gegenüber brieflich geltend gemacht, das sei ein politisches Programm, für welches keine berufliche Vereinigung, s cndern die politischen Parteien der richtige Ansatzpunkt seien. Das mag so sein. Wir dürfen aber von den politischen Parteien nicht erwarten, daß sie ohne jeden Anstoß aus der Anwaltschaft zu handeln beginnen.

Ein weiterer Kollege hat mir geschrieben, die Zusammenfassung der engagierten, freiheitsbewußten, fortschrittlichen Anwälte sei auf parteipolitischem Felde vergleichbar der Abspaltung eines Flügels von der SPD. Aber das Hinken des Vergleichs ist offenkundig: vergleichbar wäre es allenfalls der Aufspaltung einer monopolistischen bundesdeutschen Einheitspartei. Wir taten bisher so, als gebe es in einer pluralistischen Gesellschaft zwar überall Meinungsvielfalt. Nur die Anwaltschaft spreche mit einer Zunge und lebe noch sozusagen unter einem einheitlichen, geschlossenen, harmonieblauen Wertehimmel. Jedoch ist das eine wirklichkeitsferne Fiktion. Denn wie es in unserer Gesellschaft zwei annähernd gleichstarke Tendenzen gibt, eine vergangenheitskonservierende, die nicht frei von obrigkeitsstaatlichen Traditionen ist, und eine eher freiheits- und reformgeneigte, so gibt es zweifellos eine engagierte Minderheit in der Anwaltschaft, welche die für eine sinnvolle, funktionale Berufstätigkeit unentbehrlichen Freiheitsräume gegenüber der Staatsgewalt bewahren, zurückgewinnen oder erkämpfen 
will. Diese findet infolge der Mehrheitsverhältnisse im DAV dort kein Forum. Sie zusammenzufassen, ist Zweck unserer Aktion.

Und schon als sie anlief, scheint sie mir eine Wirkung wie ein Hecht im Karpfenteich gehabt zu haben: in der Stellungnahme des DAV z. B. an das Bundesverfassungsgericht im Falle Gildemaier erklangen plötzlich neue Töne: die verfaßte Anwaltschaft, die soeben noch debattierte, ob man nicht eine beamtenrechtähnliche politische Zuverlässigkeitsklausel für Rechtsanwälte haben müsse, erklärte nun plötzlich, die Zugehörigkeit zur KPD (neu) dürfe keinen Anlaß zu Disziplinarmaßnahmen gegen einen Rechtsanwalt geben. In gleicher Richtung liegt die klammheimliche - erst durch richterliche Indiskretion bekanntgewordene - Ubernahme von Verfahrenskosten durch den DAV, die den konsequent die körperliche Durchsuchung ablehnenden Rechtsanwälten Bahr-Jendges, Frommann, Heldmann und Kreischer durch Beschluß des Schwurgerichts Heidelberg auferlegt worden waren. Dergleichen wäre ohne eine sich formierende Oppositionsvereinigung schwerlich vorstellbar gewesen. Wir müssen zum Heil der Allgemeinheit ebenso konsequent unseren Weg weiterverfolgen, wie auch der konservative DAV fortfahren mag. Konservativ zu sein, ist sicherlich eine genau so ehrenhafte Haltung wie die unsere. Kurz- und mittelfristig wird wohl der Konservatismus seinen Erfolg, wird der DAV die größere Zahl - eine große Menge von Karteileichen eingerechnet - hinter sich haben. Aber langfristig lassen sich überfällige Veränderungen nicht aufhalten.*

Der Name der neuen Anwaltsvereinigung "Republikanischer Anwaltsverein " soll an den "Republikanischen Richterbund « erinnern, die Vereinigung der damals wenigen Juristen, die in der Weimarer Republik gegen die Masse ihrer noch geistig und politisch im Kaiserreich beheimateten Berufsgenossen demokratische Prinzipien verfochten. Die neue Vereinigung steht nicht nur Anwälten und Notaren, sondern auch Hochschullehrern an rechtswissenschaftlichen Fakultäten sowie Juristen in der Ausbildung offen.

Der einstimmig beschlossene Vereinigungszweck lautet in der Präambel zur Satzung u. a.:

"Die Vereinigung stellt sich in die Tradition des Kampfes um die freie Advokatur und um ein demokratisches Recht. Recht ist Instrument der Begründung und Sicherung von Herrschaft. Es ist aber auch eine Waffe, sich gegen Herrschaft zur Wehr zu setzen. Das Recht in dieser Weise zugunsten des Schwächeren zu nutzen und zu entwickeln, ist Ziel der Vereinigung. Aber nicht nur staatliche Gewalt, sondern auch gesellschaftliche und wirtschaftliche Macht bedient sich der Form des Rechts. Wichtige Aufgabe des Anwalts ist es auch hier, die Interessen wirtschaftlich und sozial Schwacher wahrzunehmen, so vor allem auf den Gebieten des Arbeitsrechts, Mietrechts, Familienrechts, des Rechts der Allgemeinen Geschäftsbedingungen usw.

Abwehr von Herrschaftsansprüchen ist auch das Eintreten für das Recht der kommenden Generationen, eine lebenswerte Existenz in einer unzerstörten Umwelt vorzufinden.

Um diese Aufgaben wahrnehmen zu können, bedarf es einer freien Anwaltschaft, die ihren Beruf frei von jeglicher, insbesondere staatlicher Bevormundung ausüben kann. Der Rechtsanwalt ist einseitig gebundener Interessenvertreter seines Mandanten und ausschließlich diesem und sich selbst verantwortlich.

In den Vorstand wurden gewählt als Vorsitzender Holtfort (SPD), als sein Vertreter RA. Hannover, ferner Dagmar Driest, Eschen, Groenewold, Husmann (CDU), Preuß, Schily und Wächtler. Schon zuvor hatte eine gewisse Presse kritisiert, die neue Vereinigung sei ein Kartell aus den Terroristen-Prozessen und deren Umfeld: Heinrich Hannover, Otto Schily, Kurt Groenwold und Anhang (DIE WELT vom 2. 2. 79). Für Demokraten liegt die Perfidie dieser Kritik auf der Hand: auch Terroristen haben einen Anspruch auf ein faires Verfahren und auf eine nachdrückliche und sachgerechte Strafverteidigung. Außer Groenewold hat man niemanden der Genannten etwas am Zeuge flicken können. Groenewold ist am 10. 7. 1978 vom Strafsenat des OLG Hamburg zu zwei Jahren Freiheitsstrafe mit Bewährung verurteilt worden, weil er nach Meinung des Gerichtes die - fließenden - Grenzen der Strafverteidigung rechtsirrig überschritten habe. Aus den schriftlichen Urteils- 
gründen (abgedruckt Kritische Justiz 1979, S. 72 mit Anmerkung von Jürgen Seifert):

„Der Senat konnte es auch nicht unberücksichtigt lassen, daß die Beiträge, deren Verbreitung als strafbare Unterstützung einer kriminellen Vereinigung gewürdigt wurden, nur ein geringer Teil der Schriftwerke sind, die der Angeklagte zur Verteidigung seiner Mandanten erlaubtermaßen verteilt hat. Von diesem gewaltigen Einsatz des Angeklagten für seine Mandanten war der Großteil rechtmäßig . . . Er befand sich bei der Verteidigung seiner ehemaligen Mandanten in einer Ausnahmesituation. Er sah, daß deren Verurteilung von der Offentlichkeit und den Massenmedien gefordert wurde und fühlte sich, um diesem Verurteilungsdruck zu begegnen, mit Recht zu besonderer Aktivität veranlaßt ....

Mit dieser Verurteilung muß die Sache ausgestanden sein. In einer freiheitlichen und solidarischen Gesellschaft darf man niemandem entgegen einem richterlichen Urteil auf »kaltem Wege« die bürgerlichen Ehrenrechte entziehen.

Die Bundesrepublik Deutschland soll nach dem Auftrage ihrer Verfassung ein freiheitlicher und sozialer Rechtsstaat sein. Manche Bürger glauben, schon zuviel davon zu haben, andere, dieses Ziel schon erreicht zu haben. Der "Republikanische Anwaltsverein « hingegen sieht in diesem Verfassungsauftrag eine ständige, immer erst zu verwirklichende Aufgabe. Es ist erwünscht, daß alle liberalen und progressiven Anwälte, Hochschullehrer, Referendare und Rechtspraktikanten eintreten. (Republikanischer Anwaltsverein e. V., Hohenzollernstr. 7, 3000 Hannover I).

Hela Rischmüller-Pörtner

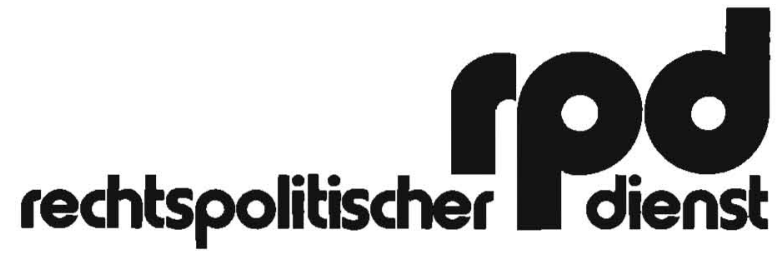

berichtet jede Woche aktuell aus Bundestag und Bundesrat, informiert über den neuesten Stand der Gesetzgebung, über wichtige Reformvorhaben und Novellierungsvorschläge, berichtet über rechtspolitisch interessante Gerichtsund Verwaltungsentscheidungen, über neue Entwicklungen im internationalen Recht, liefert die notwendigen Hintergrundinformationen und ermöglicht einen schnellen Überblick über die verschiedenen rechtspolitischen Positionen.

Auf Wunsch senden wir Ihnen den "rechtspolitischen dienst « kostenlos zur Probe. Rufen Sie inn noch heute bei uns ab.

\section{pressebüro ralf theurer}

